\title{
Demersal fish assemblages of the Gulf of Salamanca, Colombia (southern Caribbean Sea)
}

\author{
Camilo B. García*, Luis Orlando Duarte, Dominique von Schiller \\ Instituto de Investigaciones Marinas y Costeras, INVEMAR, A.A. 1016, Santa Marta, Colombia
}

\begin{abstract}
The structure of the demersal fish assemblages of the Gulf of Salamanca, Colombian Caribbean Sea, was studied based on 4 trawl surveys carried out in December 1995. April and August 1996, and February 1997. Multivariate analysis revealed the presence of 2 distinct assemblages, one belonging to the continental shelf $(<50 \mathrm{~m}$ depth) and the other belonging to the upper slope $1>50 \mathrm{~m}$ depth). The continental shelf assemblage showed significantly more biomass per tow but not more individuals or species. It is typified by species of interest for the artisanal fishery, like Lutjanus analis and Calamus penna. Lutjanus synagris, however, also of interest for the fishery, typifies the upper slope assemblage. No temporal change was detected in the assemblage structure, at least for the sampling dates in this study, but many species in the assemblages were scarce and rare, indicating high spatial and temporal patchiness. The assemblages found do not fully adjust to existing zonation schemes or assemblages defined in neighboring areas, probably due to the effect of the spatial scale of the studies and the width of the shelf in the Gulf of Salamanca.
\end{abstract}

KEY WORDS: Demersal fish assemblages Southern Caribbean Sea Colombia

\section{INTRODUCTION}

The reduction of catch rates and mean size of individuals is a well documented trend in world fisheries (Pitcher 1996). This situation has prompted new approaches to the study of exploited populations, including the study of the fish assemblage structure in relation to environmental variables, and the characterization of seasonal changes for the improvement of management practices (Cady \& Sharp 1986)

The present work originated from a similar motivation. The Gulf of Salamanca is used by the artisanal fishery with few restrictions or regulations. Recent trends of reduction of catches by the artisanal fishery on the Caribbean coast of Colombia in the last decade (5886 tonnes landed in 1986 vs 4616 landed in 1995 , INPA 1995) have made clear the need for implementing some management measurements, if the fishery is to be sustained and to have any economic importance. For the Gulf of Salamanca, an expansion of the arti-

\footnotetext{
·E-mail: cgarcia@invemar.org.co
}

sanal fishery depth range (now within the 0 to $20 \mathrm{~m}$ depth limit) has been proposed.

The study area is also of interest in ecological terms as it shows the potential for strong seasonality as a result of the wind regime that probably causes an upwelling phenomenon in interplay with the rainfall patterns which drive the seasonal entry of freshwater masses to certain areas in the gulf (see 'Study area'). The comparisons of this tropical shelf system with other tropical systems under a similar dynamic pattern might reveal some general trends in the ecology of demersal fish communities

To our knowledge, there are few multivariate studies of tropical demersal fish assemblages in the Caribbean (excluding coral reef areas, hard banks and coastal lagoons). The study done by Yañes-Arancibia \& Sánchez-Gil (1986) can be cited in this context. LoweMcConnell (1962) studied the demersal fish fauna off Guyana and Bianchi (1992a) did the same for Guyana, Surinam and Venezuela. Multivariate studies in the south Atlantic Bight of the United States have been conducted by Sedberry \& van Dolah (1984) and Wenner \& Sedberry (1989). The tropical Pacific coast of 
America has been studied by Bartels et al. (1983), Bianchi (1991) and Wolff (1996).

Studies done at several latitudes have highlighted patterns of spatial and temporal variation in composition, abundance and distribution of demersal fish assemblages. These variations have been related to gradients and fluctuations of factors such as depth (Bartels et al. 1983, Bianchi 1991, Gray \& Otway 1994), seasonal changes in water salinity and temperature (Allen 1982, Blaber et al. 1989, Watson et al. 1990, Ansari et al. 1995), distance to the coastline (Rainer \& Munro 1982), bottom type (Martin et al. 1985) and hydrographic conditions (Bianchi 1991). However, the dominant gradient affecting the distribution of the demersal fish assemblages appears to be depth.

\section{STUDY AREA}

The Gulf of Salamanca (Fig. 1) is within $11^{\circ} 00^{\prime}$ and $11^{\circ} 19^{\prime} \mathrm{N}$ and $74^{\circ} 12^{\prime}$ and $74^{\circ} 50 \mathrm{~W}$. The sampled area corresponds to the trawlable area of the gulf between the 10 and $200 \mathrm{~m}$ isobaths (Fig. 1) which accounts for $650 \mathrm{~km}^{2}$, approximately. The trawlable area was defined according to a previous bottom survey performed by the authors and previous trawl experiences
(Blanco 1993). Depths less than $10 \mathrm{~m}$ were not sampled as they were not accessible to the ships used. Fig. 2 shows the bathymetric profiles of 4 selected sectors in the gulf. As can be seen the continental shelf in the region shows a discrete size with a maximum width of just $16 \mathrm{~km}$ and the shelf slope is steep. Stromme \& Saetersdal (1989) placed the border of the Colombian Caribbean continental shelf between 50 and $60 \mathrm{~m}$ depth in average as is the case in the Gulf of Salamanca (Fig. 2).

The shelf is dominated by litoclastic sand at shallow depths but it becomes muddy toward the shelf edge (Molina 1993) and to the west in the influence area of the Magdalena river (Fig. 1, pers. obs.). In front of the Cienaga Grande de Santa Marta (Fig. 1), fine sandshell bottoms are dominant. Near the shelf edge a reeflike formation called Las Animas Bank (Blanco et al. 1994) is located, which provides spatial heterogeneity to the sea floor of the gulf but reduces the trawlable area.

The region is dry with an accumulated yearly precipitation that ranges between 400 and $800 \mathrm{~mm}$ (Eslava et al. 1986). Rainfall has a bimodal behavior (Fig. 3) generated by the interplay of the trade winds and the displacement of the Intertropical Convergence Zone (ITCZ). Between December and April, there is a

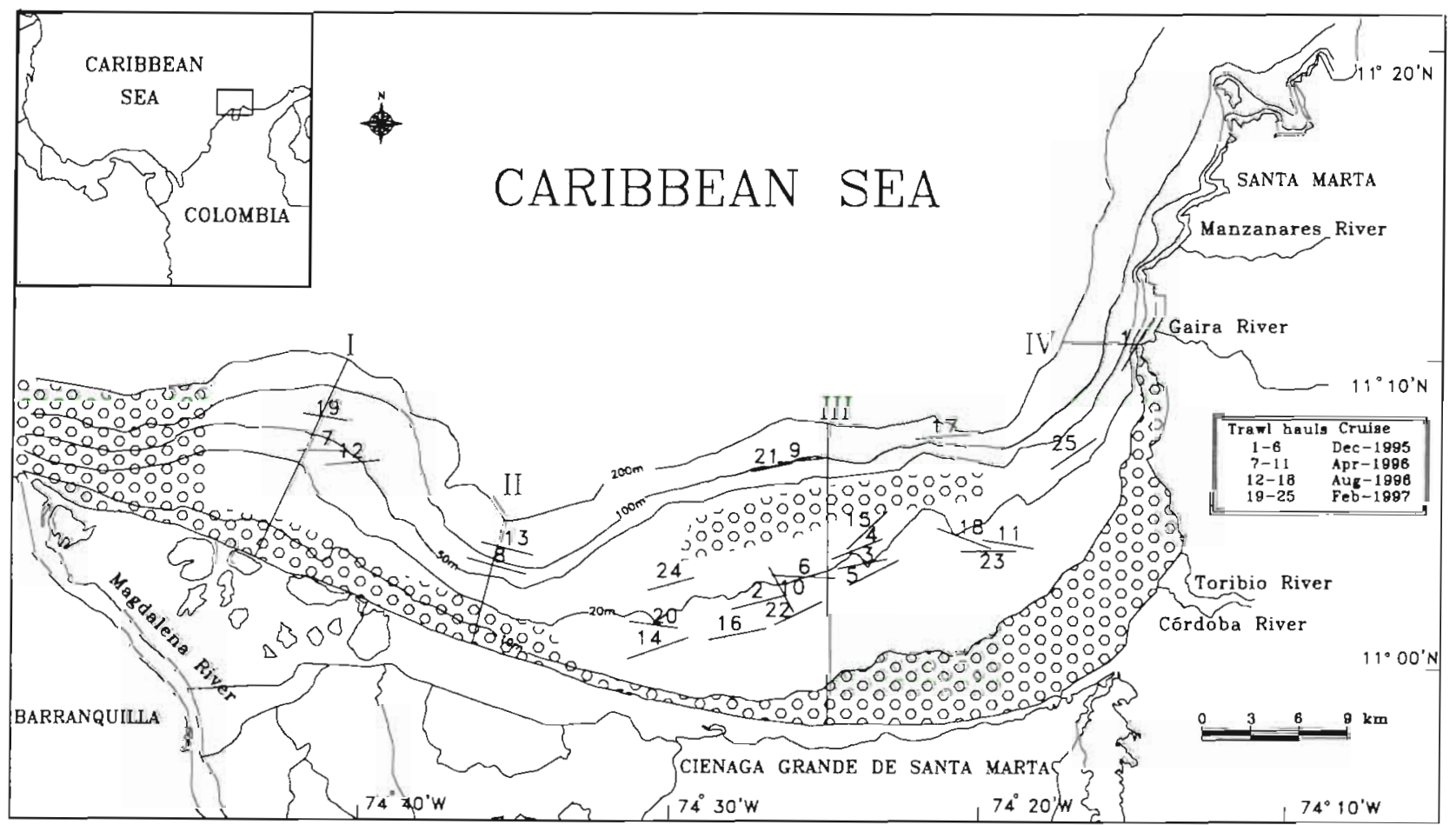

Fig. 1. The Gulf of Salamanca (Colombian Caribbean), showing sampling sites, trawlable area and the non trawlable or not accessible area (shaded), the path of each trawl and the position of the depth transects shown in Fig. 2. The area shacled less than $10 \mathrm{~m}$ depth is not accessible to the ships. The non trawlable areas are so due to permanent rough sea conditions (to the east of the Magdalena river) or due to irregular, rough bottom 
Sector I

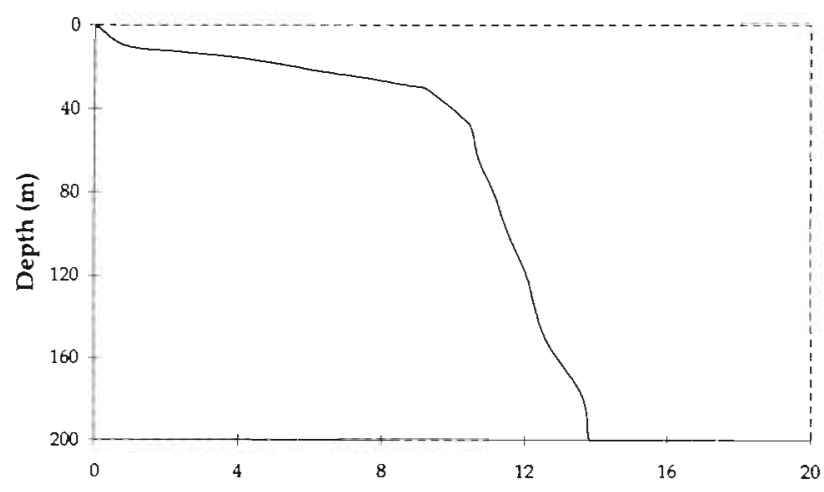

Sector III

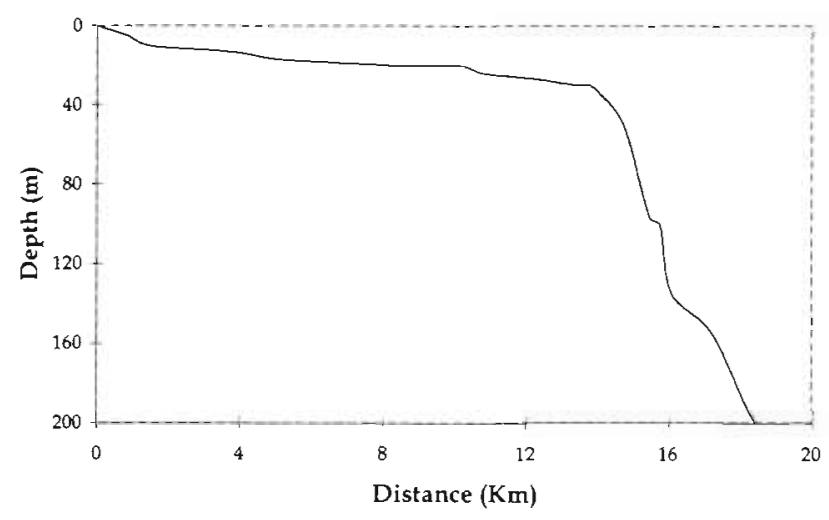

Sector II

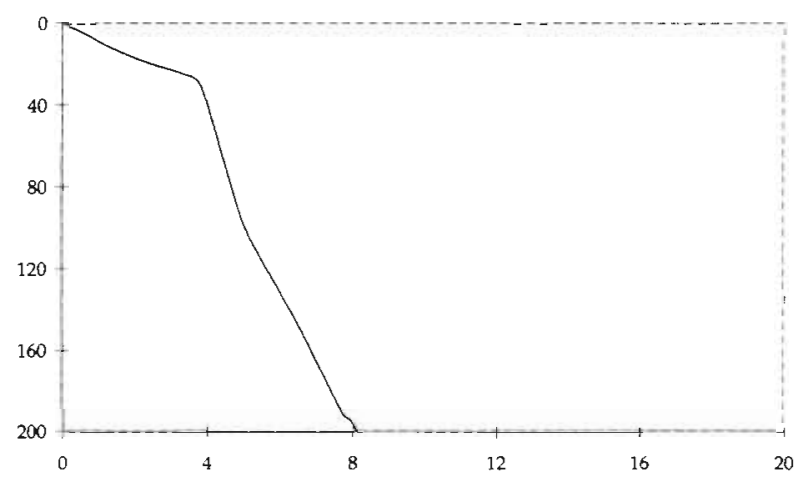

Sector IV

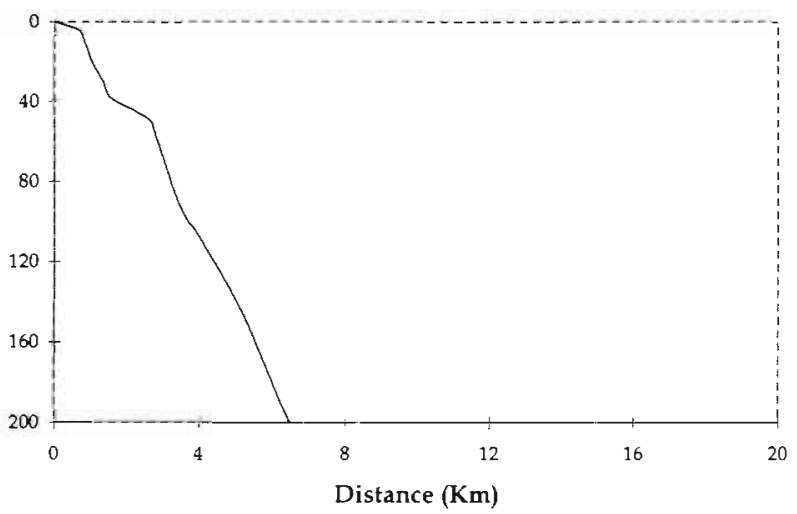

Fig. 2. Depth transects in 4 sectors of the Gulf of Salamanca (Colombian Caribbean) according to nautical chart COL-407

particularly dry period under the direct influence of the trade winds when the ITCZ occupies its southernmost position. In May and June the first rainy period occurs when atmospheric pressure is low, followed by a short dry period called 'veranillo de San Juan' in July-August. The year ends with a period (September to November) of stronger precipitation when the ITCZ is over the region (Marquez 1982, Salzwedel \& Müller 1983). Fig. 3 shows that 1996, when most of the sampling campaigns took place, was rainier than average.

The current regime is dependent upon the trade winds. When they are strong (dry season, December to April) they generate the Caribbean Current that runs southwest parallel to the coast. When the trade winds
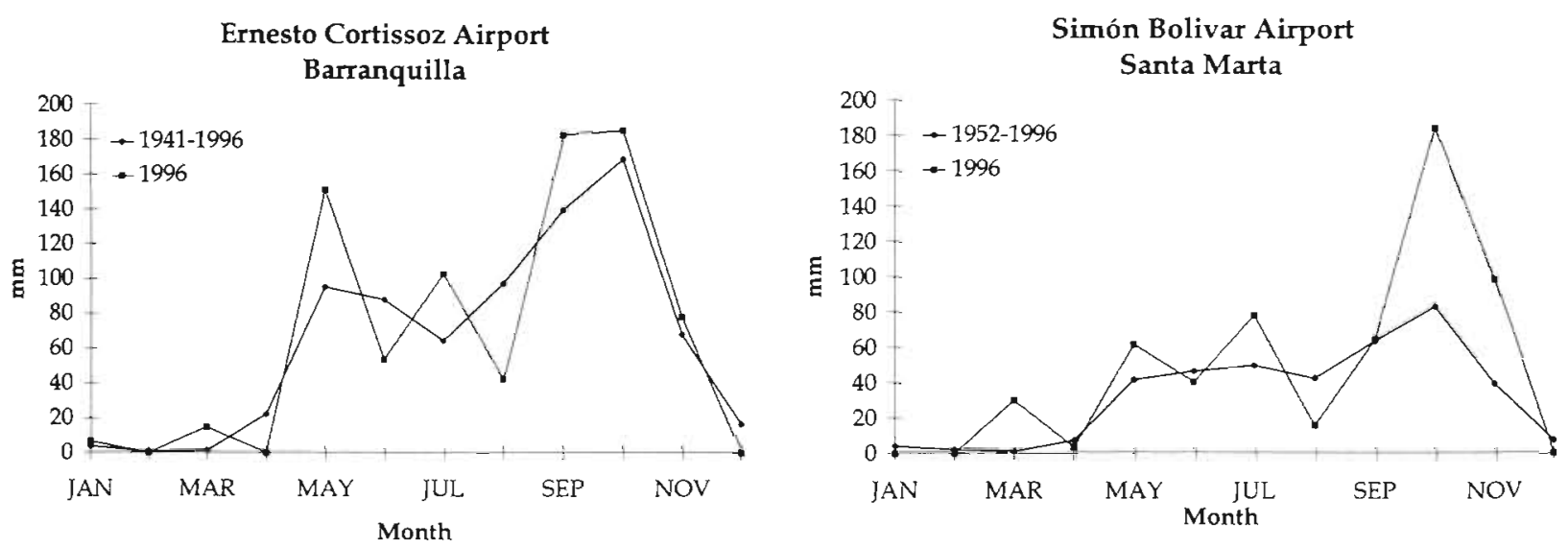

Fig. 3. Multiannual monthly patterns of precipitation and monthly precipitation in the year 1996 as registered at airports in the vicinity to the study area. Source: Instituto de Estudios Ambientales, IDEAM 
weaken (rainy season, May to November), the 'Panama' Counter Current, running northeast, eventually reaches the Guajira Peninsula $\left(12^{\circ} \mathrm{N}\right.$, Marquez 1982. Molina 1993) but because of the east-west orientation of the Gulf of Salamanca, it presumably does not enter the gulf. As a consequence of this current regime the central part of the Gulf of Salamanca presents oceanic conditions, whereas the western part is seasonally influenced by the Magdalena river plume and the east part is influenced by the Cienaga Grande de Santa Marta and the inflow of other minor rivers. Upwelling waters have been detected up to $75^{\circ} \mathrm{W}$, which includes the Gulf of Salamanca, but these waters have been characterized as oligotrophic, causing physical (water cooling) rather than biological effects (Ramirez 1993, Blanco et al, 1994).

\section{MATERIALS AND METHODS}

Trawl data. The material was collected on 4 cruises: Cruise 9600 (17 to 19 December 1995, 6 trawls) was done with the RV 'Ancon', Cruises 9601 (1.0 to 13 April 1996, 5 trawls) and 9602 (8 to 13 August 1996, 7 trawls) were done with the RV 'Malpelo' and finally cruise 9701 (6 to 9 February 1997, 7 trawls) was done again with the RV 'Ancon' For the purposes of this study the February and April cruises were considered representative of the dry season, and the August and December ones representative of the rainy season.

Table 1 shows the characteristics of the fish otter trawls used during the study. Each tow lasted 30 min at an average speed of 3.5 knots. As the number of stations per cruise was limited by the available ship time, the trawlable area (Fig. 1) was divided into 4 subareas around the depth profiles shown in Fig. 1, so as to ensure a complete cover of the Gulf, with the exception of Cruise 9600 where simple random positioning of the trawl stations resulted in an uneven cover (Fig. 1). The actual position of the stations inside a subarea was chosen randomly. Each specimen caught was counted and weighed (wet weight) separately. The biomass

Table 1 Fishing gear characteristics of the survey ships used in the study

\begin{tabular}{|lcc|}
\hline & RV 'Ancón' & RV 'Malpelo' \\
\hline Headrope $(\mathrm{m})$ & 20.60 & 33.30 \\
Groundrope $(\mathrm{m})$ & 25.60 & 41.60 \\
Trawl length $(\mathrm{m})$ & 28.50 & 38.20 \\
Bridles $(\mathrm{m})$ & 50.20 & 60.00 \\
Mesh size $(\mathrm{mm})$ & 45.00 & 50.80 \\
Mean path width $(\mathrm{m})$ & 11.14 & 19.03 \\
\hline
\end{tabular}

and density were estimated by means of the 'swept area' method (Espino \& Wosnitza-Mendo 1984). For this calculation, divergence measurements between the 2 cables of the net were done at regular intervals 5 times during each tow. The mean swept area for a trawl in the study was $0.04381 \mathrm{~km}^{2}$, to which biomass and numbers of individuals are referred.

At each station water column and bottom salinity and temperature were measured and sediment samples were taken by means of a van Veen grab. In Cruise 9602, the water temperature was not measured due to a malfunction of the CTD probe.

Data analysis. Since the main purpose of this study was to identify the assemblages of demersal fishes and to relate them to environmental factors, the analysis strategy described by Field et al. (1982) was followed. The trawl stations were ordered on the basis of a biomass matrix by means of a nonmetric multidimensional scaling (NMDS) ordination. The typifying and discriminating species of the clusters of stations revealed by the NMDS were determined using the SIMPER procedure (Clarke 1993). This procedure determines the average contribution of each species to the similarity (typifying species) and dissimilarity (discriminating species) between groups of samples (Clarke 1993).

A relationship to environmental data was obtained by superimposing the environmental data as symbols scaled in size to the data value on the biotic NMDS ordination (Field et al. 1982) and by the BIO-ENV procedure proposed by Clarke \& Ainsworth (1993). This procedure measures the match between the similarity matrices underlying both biotic and abiotic NMDS ordination of the samples by means of a rank correlation (Spearman) coefficient (Clarke \& Ainsworth 1993).

In order to test the null hypothesis that the demersal fish assemblages do not differ in structure between depth strata, the ANOSIM procedure (Clarke \& Green 1988. Clarke 1993) was used. This test is based on the rank similarities between samples in the underlying similarity matrix. A test statistic $R$ is defined as the difference between the average of all rank similarities among replicate samples within groups, and the average of rank similarities arising from all pairs of samples between groups, the groups of samples defined under the null hypothesis. The $R$ statistic is recomputed under permutation of the sample labels. The significance level of the test is found by referring the observed $R$ value to its permutation distribution. If the observed $R$ value looks unlikely to have come from this distribution the null hypothesis can be rejected at a significance level of $100(t+1) /(T+1) \%$, where $t$ is the number of the $T$ simulated $R$ values as large as or larger than the observed $R$ (Clarke \& Green 1988, Clarke 1993).

By means of 1 -way ANOVAs, the null hypothesis that Hill diversity numbers 1 and 2 (Hill 1973), species 
richness, density and biomass per trawl station do not differ between depth strata was tested. In equation form Hill's family of diversity numbers are:

$$
\mathrm{NA}=\sum_{i=1}^{s}\left(p_{i}\right)^{1 /(1-A)}
$$

where $p_{1}$ is the proportion of biomass (in this case) belonging to the $i$ th species, $A$ is the order of these diversity numbers, and $s$ is the number of species.

Biomass dominance patterns were compared between depth strata by means of $\mathrm{k}$-dominance curves (Lambshead et al. 1983) which represent the ranking in percentage of the species in decreasing order of importance. Logging the $x$ (rank) axis allows better visualization of the plot.

The temporal fish assemblage change was tested by the following null hypotheses: the assemblage structure between cruises is not different (ANOSIM procedure, Clarke \& Green 1988, Clarke 1993), in this case with the trawl stations grouped by depth stratum in order to control for this factor, and Hill diversity numbers 1 and 2 (Hill 1973), species richness, density and biomass per trawl station do not differ between cruises, in this case regardless of depth stratum.

For all the ANOVA tests normality of the data and homogeneity of variance were tested and data were transformed (number of individuals and biomass, $\log [x+1])$ when necessary. In case of a significant $F$, a multiple comparisons test was performed (Tukey test, Zar 1984).

\section{RESULTS}

Table 2 shows the geographical location, date, time and environmental values of each trawl station. Trawling covered depths between 13 and $154 \mathrm{~m}$. Because the area with depths between 10 and $30 \mathrm{~m}$ represented more than $50 \%$ of the trawlable area, sampling was concentrated there.

From the 25 trawl stations a total of 6680 individuals weighing $1873.4 \mathrm{~kg}$ was collected. A total of 126 species was found distributed in 54 families. Table 3 shows the ranking of these species according to their frequency, accumulated abundance and biomass. A feature of the presence-dominance structure of this demersal fish community was the occurrence of a small group of species that were common and abundant in numbers and biomass throughout the gulf. This background group (defined as those species that appeared at least 3 times and their number or biomass ranked among the first 10, Table 3) included species like Lutjanus analis, Lutjanus synagris, Calamus penna, Balistes capriscus, Selene vomer, Selene setapinnis, Diodon holacanthus, Eucinostomus argenteus, Chloro-

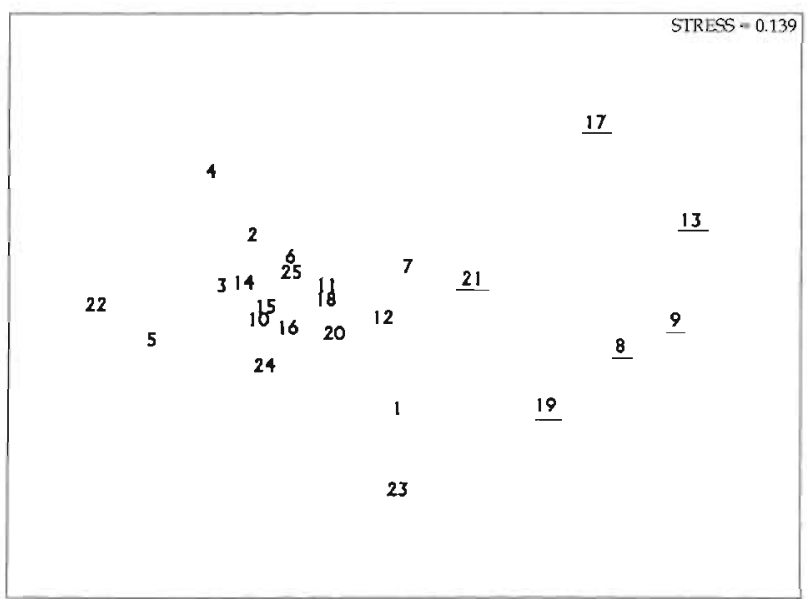

Fig. 4. Ordination (NMDS) of the trawl stations in the Gulf of Salamanca (Colombian Caribbean). Underlined trawl station numbers are stations placed deeper than $50 \mathrm{~m}$
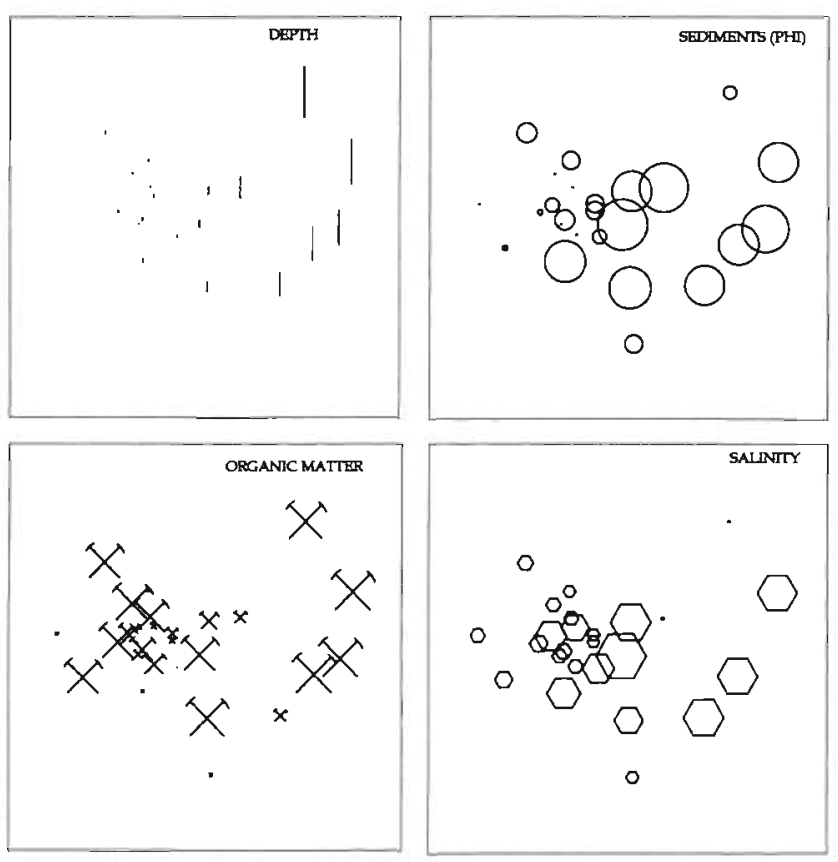

Fig. 5. Superposition of some environmental variables on the trawl stations ordination shown in Fig. 4. Symbol size is in proportion to variable value

scombrus chrysurus, Lutjanus jocu, Eucinostomus gula, Sciaena trewavasae and Holocentrus ascensionis. The remaining 113 species are scarce or rare (Table 3).

Fig. 4 shows the NMDS ordination of the 25 stations of the study. Two clusters of stations along the $x$ axis can be distinguished. Superimposition of the environmental variables (Fig. 5) and the BIO-ENV analysis (Table 4) reveals that depth and, secondarily, median grain size (phi transformation, Buchanan \& Kain 1984) 
Table 2. Geographical position and environmental variable values of the trawl stations in the Gulf of Salamanca, Colombian Caribbean

\begin{tabular}{|c|c|c|c|c|c|c|c|}
\hline Cruise 9600 & $\operatorname{stn} 1$ & Stn 2 & Stn 3 & Stn 4 & $\operatorname{Stn} 5$ & Stn 6 & \\
\hline Date $(\mathrm{mm} / \mathrm{dd} / \mathrm{yy})$ & $12 / 16 / 95$ & $12 / 18 / 95$ & $12 / 18 / 95$ & $12 / 19 / 95$ & $12 / 19 / 95$ & $12 / 19 / 95$ & \\
\hline Initial time (h) & $11: 00$ & $13: 30$ & $16: 15$ & $09: 10$ & $14: 05$ & $15: 18$ & \\
\hline Final time (h) & $11: 30$ & $14: 00$ & $16: 47$ & $09: 40$ & $14: 37$ & $15: 50$ & \\
\hline Coordinates, initial: $\begin{array}{rl}N & 1 \\
W & 7\end{array}$ & $\begin{array}{l}11^{\circ} 09.900^{\prime} \\
74^{\circ} 15.500^{\prime}\end{array}$ & $\begin{array}{l}11^{\circ} 03.580^{\prime} \\
74^{\circ} 26.310^{\prime}\end{array}$ & $\begin{array}{l}11^{\circ} 04.430^{\prime} \\
74^{\circ} 24.680^{\prime}\end{array}$ & $\begin{array}{l}11^{\circ} 05.400^{\prime} \\
74^{\circ} 23.240^{\prime}\end{array}$ & $\begin{array}{l}11^{\circ} 05.120^{\prime} \\
74^{\circ} 22.830^{\prime}\end{array}$ & $\begin{array}{l}11^{\circ} 04.210^{\prime} \\
74^{\circ} 26.790^{\prime}\end{array}$ & \\
\hline Coordinates, final: $\underset{W}{\mathrm{~N}}$ & $\begin{array}{l}11^{\circ} 11.430^{\prime} \\
74^{\circ} 14.620^{\prime}\end{array}$ & $\begin{array}{l}11^{\circ} 03.120^{\prime} \\
74^{\circ} 28.090^{\prime}\end{array}$ & $\begin{array}{l}11^{\circ} 04.750^{\prime} \\
74^{\circ} 23.100^{\prime}\end{array}$ & $\begin{array}{l}11^{\circ} 04.740^{\prime} \\
74^{\circ} 24.880^{\prime}\end{array}$ & $\begin{array}{l}11^{\circ} 03.900^{\prime} \\
74^{\circ} 24.300^{\prime}\end{array}$ & $\begin{array}{l}11^{\circ} 03.910^{\prime} \\
74^{\circ} 24.800^{\prime}\end{array}$ & \\
\hline Median depth (m) & 41.5 & 17.7 & 18.5 & 20.5 & 16.5 & 18.5 & \\
\hline Sediments $(\Phi)$ & 3.94 & 2.11 & 2.29 & 2.96 & 2.29 & 2.11 & \\
\hline Organic matter ( $\%$ dry wt) & t) 15.642 & 10.701 & 11.883 & 10.953 & 11.883 & 10.701 & \\
\hline Bottom salinity & 36.77 & 36.70 & 36.64 & 36.64 & 36.64 & 36.60 & \\
\hline Bottom temperature $\left({ }^{\circ} \mathrm{C}\right)$ & 25.68 & 26.38 & 26.56 & 26.36 & 26.36 & 26.60 & \\
\hline Cruise 9601 & $\operatorname{Stn} 7$ & $\operatorname{Stn} 8$ & Stn 9 & $\operatorname{stn} 10$ & $\operatorname{Stn} 11$ & & \\
\hline Date $(\mathrm{mm} / \mathrm{dd} / \mathrm{yy})$ & $4 / 10 / 96$ & $4 / 11 / 96$ & $4 / 11 / 96$ & $4 / 11 / 96$ & $4 / 12 / 96$ & & \\
\hline Initial time (h) & $20: 35$ & $11: 24$ & $20: 00$ & $06: 58$ & $16: 05$ & & \\
\hline Final time (h) & $21: 05$ & $11: 59$ & $20: 36$ & $07: 30$ & $16: 35$ & & \\
\hline 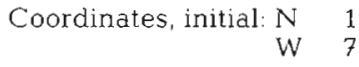 & $\begin{array}{l}11^{\circ} 07.969^{\prime} \\
74^{\circ} 40.504^{\prime}\end{array}$ & $\begin{array}{l}11^{\circ} 03.267^{\prime} \\
74^{\circ} 34.706^{\prime}\end{array}$ & $\begin{array}{l}11^{\circ} 07.043^{\prime} \\
74^{\circ} 25.166^{\prime}\end{array}$ & $\begin{array}{l}11^{\circ} 03.026^{\prime} \\
74^{\circ} 26.078^{\prime}\end{array}$ & $\begin{array}{l}11^{\circ} 04.902^{\prime} \\
74^{\circ} 20.006^{\prime}\end{array}$ & & \\
\hline Coordinates, final: $\underset{W}{N}$ & $\begin{array}{l}11^{\circ} 08.003^{\prime} \\
74^{\circ} 42.225^{\prime}\end{array}$ & $\begin{array}{l}11^{\circ} 03.789^{\prime} \\
74^{\circ} 36.635^{\prime}\end{array}$ & $\begin{array}{l}11^{\circ} 06.854^{\prime} \\
74^{\circ} 27.081^{\prime}\end{array}$ & $\begin{array}{l}11^{\circ} 04.481^{\prime} \\
74^{\circ} 26.881^{\prime}\end{array}$ & $\begin{array}{l}11^{\circ} 04.641^{\prime} \\
74^{\circ} 18.341^{\prime}\end{array}$ & & \\
\hline Median depth (m) & 32.5 & 105.5 & 109.0 & 17.0 & 13.0 & & \\
\hline Sediments $(\Phi)$ & 3.85 & 3.87 & 4.16 & 2.11 & 2.87 & & \\
\hline Organic matter ( $\%$ dry wt) & t) 19.497 & 19.283 & 15.739 & 10.701 & 9.974 & & \\
\hline Bottom salinity & 36.08 & 36.82 & 36.81 & 35.58 & 35.85 & & \\
\hline Bottom temperature $\left({ }^{\circ} \mathrm{C}\right)$ & 26.55 & 23.95 & 23.89 & 26.95 & 27.36 & & \\
\hline Cruise 9602 & $\operatorname{Stn} 12$ & $\operatorname{Stn} 13$ & Stn 14 & Stn 15 & $\operatorname{Stn} 16$ & Stn 17 & $\operatorname{Stn} 18$ \\
\hline Date $(\mathrm{mm} / \mathrm{dd} / \mathrm{yy})$ & $8 / 8 / 96$ & $8 / 9 / 96$ & $8 / 10 / 96$ & $8 / 10 / 96$ & $8 / 10 / 96$ & $8 / 13 / 96$ & $8 / 13 / 96$ \\
\hline Initial time (h) & $11: 11$ & $17: 25$ & $07: 57$ & $12: 05$ & $08: 01$ & $05: 56$ & $10: 49$ \\
\hline Final time (h) & $11: 40$ & $1.7: 55$ & $08: 33$ & $12: 35$ & $08: 32$ & $06: 25$ & $11: 19$ \\
\hline $\begin{array}{r}\text { Coordinates, initial: } N \\
W\end{array}$ & $\begin{array}{l}11^{\circ} 07.520^{\prime} \\
74^{\circ} 41.380^{\prime}\end{array}$ & $\begin{array}{l}11^{\circ} 04.250^{\prime} \\
74^{\circ} 35.820^{\prime}\end{array}$ & $\begin{array}{l}11^{\circ} 03.230^{\circ} \\
74^{\circ} 31.750^{\prime}\end{array}$ & $\begin{array}{l}11^{\circ} 04.930^{\prime} \\
74^{\circ} 24.120^{\prime}\end{array}$ & $\begin{array}{l}11^{\circ} 03.010^{\prime} \\
74^{\circ} 28.820^{\prime}\end{array}$ & $\begin{array}{l}11^{\circ} 08.010^{\prime} \\
74^{\circ} 20.490^{\prime}\end{array}$ & $\begin{array}{l}11^{\circ} 04.516^{\prime} \\
74^{\circ} 19.450^{\prime}\end{array}$ \\
\hline Coordinates, final: $\mathrm{N}$ & $\begin{array}{l}11^{\circ} 07.700^{\prime} \\
74^{\circ} 39.520^{\prime}\end{array}$ & $\begin{array}{l}11^{\circ} 03.890^{\prime} \\
74^{\circ} 34.500^{\prime}\end{array}$ & $\begin{array}{l}11^{\circ} 04.210^{\prime} \\
74^{\circ} 29.580^{\prime}\end{array}$ & $\begin{array}{l}11^{\circ} 06.300^{\prime} \\
74^{\circ} 23.090^{\prime}\end{array}$ & $\begin{array}{l}11^{\circ} 03.390^{\prime} \\
74^{\circ} 26.940^{\prime}\end{array}$ & $\begin{array}{l}11^{\circ} 07.910^{\prime} \\
74^{\circ} 22.210^{\prime}\end{array}$ & $\begin{array}{l}11^{\circ} 05.290^{\prime} \\
74^{\circ} 21.500^{\prime}\end{array}$ \\
\hline Median depth (m) & 32.0 & 137.0 & 13.5 & 22.5 & 15.0 & 153.5 & 13.3 \\
\hline Sediments $(\Phi)$ & 4.32 & 3.83 & 2.70 & 2.96 & 2.11 & 2.65 & 2.87 \\
\hline Organic matter ( $\%$ dry wt $)$ & t) 22.843 & 19.232 & 16.692 & 11.405 & 10.701 & 6.289 & 9.974 \\
\hline Bottom salinity & 36.64 & 36.81 & 36.19 & 36.28 & 36.19 & 36.71 & 35.70 \\
\hline Botton temperature $\left({ }^{\circ} \mathrm{C}\right)$ & - & - & - & - & - & - & - \\
\hline Cruise 9701 & Stn 19 & Str 20 & $\operatorname{Stn} 21$ & $\operatorname{stn} 22$ & $\operatorname{Stn} 23$ & $\operatorname{Stn} 24$ & $\operatorname{Sin} 25$ \\
\hline Date $(\mathrm{mm} / \mathrm{dd} / \mathrm{yy})$ & $2 / 6 / 97$ & $2 / 7 / 97$ & $2 / 8 / 97$ & $2 / 8 / 97$ & $2 / 9 / 97$ & $2 / 15 / 97$ & $2 / 19 / 97$ \\
\hline Initial time (h) & $08: 18$ & $17: 44$ & $11: 40$ & $16: 09$ & $09: 35$ & $09: 18$ & $11: 28$ \\
\hline Final time $(\mathrm{h})$ & $08: 48$ & $18: 15$ & $12: 13$ & $16: 40$ & $10: 05$ & $09: 48$ & $12: 04$ \\
\hline $\begin{array}{r}\text { Coordinates, initial: } \mathrm{N} \\
\mathrm{W}\end{array}$ & $\begin{array}{l}11^{\circ} 09.201^{\prime} \\
74^{\circ} 42.005^{\prime}\end{array}$ & $\begin{array}{l}11^{\circ} 03.399^{\prime} \\
74^{\circ} 31.498^{\prime}\end{array}$ & $\begin{array}{l}11^{\circ} 06.605^{\prime} \\
74^{\circ} 27.482^{\prime}\end{array}$ & $\begin{array}{l}11^{\circ} 03.235^{\prime} \\
74^{\circ} 25.428^{\prime}\end{array}$ & $\begin{array}{l}11^{\circ} 04.547^{\prime} \\
74^{\circ} 18.900^{\prime}\end{array}$ & $\begin{array}{l}11^{\circ} 04.391^{\prime} \\
74^{\circ} 30.926^{\prime}\end{array}$ & $\begin{array}{l}11^{\circ} 07.189^{\prime} \\
74^{\circ} 17.794^{\prime}\end{array}$ \\
\hline Coordinates, tinal: $\underset{W}{N}$ & $\begin{array}{l}11^{\circ} 08.962^{\prime} \\
74^{\circ} 40.513^{\prime}\end{array}$ & $\begin{array}{l}11^{\circ} 03.187^{\prime} \\
74^{\circ} 29.933^{\prime}\end{array}$ & $\begin{array}{l}11^{\circ} 06.873^{\prime} \\
74^{\circ} 26.178^{\prime}\end{array}$ & $\begin{array}{l}11^{\circ} 02.583^{\prime} \\
74^{\circ} 26.656^{\prime}\end{array}$ & $\begin{array}{l}11^{\circ} 04.535^{\prime} \\
74^{\circ} 20.713^{\prime}\end{array}$ & $\begin{array}{l}11^{\circ} 04.804^{\prime} \\
74^{\circ} 29.416^{\prime}\end{array}$ & $\begin{array}{l}11^{\circ} 08.210^{\prime} \\
74^{\circ} 16.306^{\prime}\end{array}$ \\
\hline Median depth $(\mathrm{m})$ & 77.0 & 19.5 & 73.0 & 15.3 & 12.8 & 24.8 & 21.5 \\
\hline Sediments $(\Phi)$ & 3.85 & 2.70 & 4.24 & 2.11 & 2.87 & 3.92 & 2.08 \\
\hline Organic matter ( $\%$ dry wt) & t) 19.497 & 16.692 & 6.672 & 10.701 & 9.974 & 17.297 & 15.285 \\
\hline Bottom salinity & 35.90 & 35.61 & 36.10 & 35.60 & 35.62 & 35.60 & 35.75 \\
\hline Bottom temperature $\left({ }^{\circ} \mathrm{C}\right)$ & 25.60 & 26.20 & 25.80 & 26.00 & 26.10 & 26.20 & 26.40 \\
\hline
\end{tabular}


Table 3. Species list, frequency (presence in the trawl stations), total numbers and accumulated biornass (g) of the species caught in the study by means of demersal trawls in the Gulf of Salamanca, Colombian Caribbean. Superscripts indicate the top 10 ranked species in number and biomass

\begin{tabular}{|c|c|c|c|c|c|c|c|}
\hline Species & Frequency & Number & Biomass & Species & Frequency & Number & Biomass \\
\hline Lutjanus analis & 19 & 198 & $252900^{2}$ & Dasyatis guttata & 2 & 3 & 20530 \\
\hline Lutjanus synagris & 16 & $769^{2}$ & $245965^{3}$ & Pomacanthus arcuatus & 2 & 3 & 1620 \\
\hline Balistes capriscus & 15 & $870^{1}$ & $310525^{\prime}$ & Pagrus pagrus & 2 & 3 & 1620 \\
\hline Calamus penna & 15 & $247^{9}$ & $152680^{4}$ & Squatina dumeril & 2 & 3 & 1410 \\
\hline Selene vomer & 13 & 57 & 27090 & Alectis ciliaris & 2 & 2 & 1710 \\
\hline Acanthostracion poligonius & 9 & 81 & 15695 & Ocyurus chrysurus & 2 & 2 & 1200 \\
\hline Diodon holacanthus & 7 & $527^{3}$ & $140510^{5}$ & Epinephelus niveatus & 2 & 2 & 270 \\
\hline Selene setapinnis & 7 & 219 & $34955^{9}$ & Chaetodon capistratus & 2 & 2 & 90 \\
\hline Rhomboplites aurorubens & 7 & 35 & 5735 & Haemulon steindachneri & 1 & $493^{4}$ & $85960^{6}$ \\
\hline Pomacanthus paru & 7 & 15 & 18925 & Menticirrhus littoralis & 1 & 37 & 3550 \\
\hline Balistes vetula & 7 & 15 & 16120 & Diapterus rhombeus & 1 & 27 & 2420 \\
\hline Ogcocephalus cf. nasutus & 7 & 13 & 3100 & Haemulon plumieri & 1 & 20 & 10000 \\
\hline Eucinostomus argenteus & 6 & $448^{5}$ & 19490 & Caranx ruber & 1 & 11 & 4060 \\
\hline Chaetodipterus faber & 6 & 52 & 29975 & Eugerres plumieri & 1 & 11 & 2225 \\
\hline Trichiurus lepturus & 6 & 13 & 3830 & Saurida brasiliensis & 1 & 11 & 160 \\
\hline Chloroscombrus chrysurus & 5 & $306^{7}$ & 17490 & Cynoscion jamaicensis & 1 & 10 & 1200 \\
\hline Opisthonema oglinum & 5 & 36 & 4590 & Trachinotus carolinus & 1 & 8 & 13720 \\
\hline Caranx crysos & 5 & 17 & 7680 & Macrodon ancylodon & 1 & 6 & 2000 \\
\hline Acanthostracion quadricornis & 5 & 15 & 2685 & Pristipomoides macrophthalmus & 1 & 6 & 1160 \\
\hline Caranx bartholomei & 5 & 8 & 11150 & Lutjanus cyanopterus & 1 & 6 & 360 \\
\hline Anisotremus virginicus & 5 & 7 & 2060 & Mustelus cf. canis & 1 & 5 & 2500 \\
\hline Eucinostomus gula & 4 & $238^{10}$ & 9815 & Chaetodon ocellatus & 1 & 5 & 400 \\
\hline Ctenosciaena gracilicirrhus & 4 & 91 & 8530 & Calamus calamus & 1 & 4 & 2280 \\
\hline Sphyraena guachancho & 4 & 35 & 11215 & Prionotus punctatus & 1 & 4 & 400 \\
\hline Lutjanus jocu & 4 & 33 & $51215^{8}$ & Scyacium micrurum & 1 & 3 & 190 \\
\hline Rhinesomus bicaudalis & 4 & 24 & 9100 & Diapterus auratus & 1 & 2 & 950 \\
\hline Priacanthus arenatus & 4 & 5 & 2340 & Raja cervigoni & 1 & 2 & 540 \\
\hline Narcine brasiliensis & 4 & 5 & 2000 & Hemicaranx amblyrhynchus & 1 & 2 & 230 \\
\hline Scorpaena plumieri & 4 & 5 & 1795 & Prionotus stearnsi & 1 & 2 & 220 \\
\hline Sciaena trewavasae & 3 & $380^{6}$ & $34910^{10}$ & Pomadasys crocro & 1 & 2 & 160 \\
\hline Holocentrus ascensionis & 3 & $263^{8}$ & $63440^{7}$ & Diodon histrix & 1. & 1 & 5500 \\
\hline Pristipomoides aquilonaris & 3 & 142 & 11880 & Caranx falcatus & 1 & 1 & 2100 \\
\hline Micropogonias furnieri & 3 & 92 & 17460 & Rhinobatos perceles & 1 & 1 & 800 \\
\hline Haemulon aurolineatum & 3 & 73 & 3465 & Lagocephalus laevigatus & 1 & 1 & 800 \\
\hline Serranus atrobranchus & 3 & 39 & 330 & Lutjanus vivanus & 1 & 1 & 750 \\
\hline Haemulon boschmae & 3 & 33 & 6800 & Echeneis naucrates & 1 & 1 & 750 \\
\hline Albula vulpes & 3 & 26 & 13890 & Epinephelus itajara & 1 & 1 & 700 \\
\hline Lutjanus apodus & 3 & 20 & 11685 & Aluterus monoceros & 1 & 1 & 700 \\
\hline Selar crumenophthalmus & 3 & 17 & 4010 & Calamus sp. & 1 & 1 & 520 \\
\hline Upeneus parvus & 3 & 15 & 860 & Umbrina broussonnettii & 1 & 1 & 480 \\
\hline Caranx hippos & 3 & 14 & 6435 & Sparisoma rubiprinne & 1 & 1 & 460 \\
\hline Anisotremus surinamensis & 3 & 13 & 10675 & Trachinotus goodei & 1 & 1 & 450 \\
\hline Acanthurus chirurgus & 3 & 12 & 5200 & Caulolatilus guppyi & 1 & 1 & 420 \\
\hline Trachinotus falcatus & 3 & 9 & 16045 & Gymnothorax ocellatus & 1 & 1 & 300 \\
\hline Fistularia tabacaria & 3 & 9 & 3070 & Prionotus roseus & 1 & 1 & 220 \\
\hline Scomberomorus brasiliensis & 3 & 8 & 3030 & Synodus foetens & 1 & 1 & 200 \\
\hline Bagre marinus & 3 & 8 & 1690 & Scorpaena brasiliensis & 1 & 1 & 200 \\
\hline Saurida normani & 3 & 8 & 730 & Polydactylus sp. & 1 & 1 & 200 \\
\hline Lutjanus purpureus & 3 & 6 & 6960 & Acanthurus bahianus & 1 & 1 & 200 \\
\hline Fistularia petimba & 3 & 6 & 1860 & Haemulon chrysargyreum & 1 & 1 & 120 \\
\hline Steindachneria argentea & 2 & 154 & 1340 & Dactylopterus volitans & 1 & 1 & 120 \\
\hline Porichthys plectrodon & 2 & 86 & 1190 & Larimus breviceps & 1 & 1 & 80 \\
\hline Selene brownii & 2 & 22 & 3205 & Cynoponticus savanna & 1 & 1 & 80 \\
\hline Synagrops trispinosus & 2 & 16 & 285 & Prionotus arenatus & 1 & 1 & 40 \\
\hline Calamus pennatula & 2 & 15 & 9745 & Lutjanus bucanella & 1 & 1 & 30 \\
\hline Albula nemoptera & 2 & 11 & 4760 & Apogon quadrisquamatus & 1 & 1 & 25 \\
\hline Diaphus cf. dumerili & 2 & 11 & 30 & Paralepididae & 1 & 1 & 20 \\
\hline Neoepinnula orientalis & 2 & 6 & 450 & Lepophidium aporthox & 1 & 1 & 20 \\
\hline Lutjanus mahogoni & 2 & 5 & 900 & Synodus poeyi & 1 & 1 & 15 \\
\hline Eucinostomus sp. & 2 & 5 & 220 & Cytharichthys of dinoceros & 1 & 1 & 10 \\
\hline Dasyatis americana & 2 & 4 & 5810 & Chaetodon sedentarius & 1 & 1 & 10 \\
\hline Haemulon album & 2 & 4 & 1940 & Paraconger sp. & 1 & 1 & 5 \\
\hline Pseudopeneus maculatus & 2 & 4 & 220 & & & & \\
\hline
\end{tabular}


Table 4. BIO-ENV combination of the environmental variables ranked according to the match of biotic and abiotic similarity matrices. 1: depth; 2 : sediment $\Phi_{i} 3$ : organic matter; 4: bottom salinity; 5 : bottom temperature; 6 : northern coordinate; 7 : western coordinate; 8 : rainfall 9 : sampling date

\begin{tabular}{|c|c|c|c|c|c|c|c|c|c|c|}
\hline & $r$ & 1 & 2 & 3 & 4 & 5 & 6 & 7 & 8 & 9 \\
\hline \multicolumn{11}{|c|}{ Cruise 9600} \\
\hline 1 & 0.804 & $\mathrm{x}$ & & & & & & & & \\
\hline 2 & 0.797 & $\mathrm{x}$ & $x$ & & & & & & & \\
\hline 3 & 0.797 & $\mathrm{x}$ & $\mathrm{x}$ & $\mathrm{x}$ & & & & & & \\
\hline 4 & 0.720 & $\mathrm{x}$ & $\mathrm{x}$ & $\mathrm{x}$ & & & $\mathrm{x}$ & & & \\
\hline 5 & 0.660 & $\mathrm{x}$ & $\mathrm{x}$ & $\mathrm{x}$ & & $x$ & $\mathrm{x}$ & & & \\
\hline 6 & 0.665 & $x$ & $\mathrm{x}$ & $\mathrm{x}$ & & $\mathrm{x}$ & $\mathrm{x}$ & $\mathrm{x}$ & & \\
\hline 7 & 0.604 & $\mathrm{x}$ & $\mathrm{x}$ & $\mathrm{x}$ & $\mathrm{x}$ & $\mathrm{x}$ & $\mathrm{x}$ & $\mathrm{x}$ & & \\
\hline \multicolumn{11}{|c|}{ Cruise 9601} \\
\hline 1 & 0.955 & $\mathrm{x}$ & & & $\mathrm{x}$ & $\mathrm{x}$ & $\mathrm{x}$ & & & \\
\hline 2 & 0.946 & $\mathrm{x}$ & $x$ & & $\mathrm{x}$ & $\mathrm{x}$ & $\mathrm{x}$ & & & \\
\hline 3 & 0.924 & $\mathrm{x}$ & & & & & & & & \\
\hline 4 & 0.924 & $\mathrm{x}$ & & & & $\mathrm{x}$ & & & & \\
\hline 5 & 0.924 & $\mathrm{x}$ & & & $\mathrm{x}$ & $\mathrm{x}$ & & & & \\
\hline 6 & 0.862 & $\mathrm{x}$ & $\mathrm{x}$ & & $\mathrm{x}$ & $\mathrm{x}$ & $\mathrm{x}$ & $\mathrm{x}$ & & \\
\hline 7 & 0.753 & $\mathrm{x}$ & $\mathrm{x}$ & $\mathrm{x}$ & $\mathrm{x}$ & $x$ & $\mathrm{x}$ & $\mathrm{x}$ & & \\
\hline \multicolumn{11}{|c|}{ Cruise 9602} \\
\hline 1 & 0.749 & $\mathrm{x}$ & & & & & & & & \\
\hline 2 & 0.694 & $\mathrm{x}$ & & & $\mathrm{x}$ & & & & & \\
\hline 3 & 0.637 & $\mathrm{x}$ & & $\mathrm{x}$ & $\mathrm{x}$ & & & & & \\
\hline 4 & 0.568 & $\mathrm{x}$ & & $\mathrm{x}$ & $\mathrm{x}$ & & $\mathrm{x}$ & & & \\
\hline 5 & 0.450 & $\mathrm{x}$ & & $\mathrm{x}$ & $x$ & & $\mathrm{x}$ & $\mathrm{x}$ & & \\
\hline 6 & 0.359 & $\mathrm{x}$ & $x$ & $\mathrm{x}$ & $\mathrm{x}$ & & $\mathrm{x}$ & $x$ & & \\
\hline \multicolumn{11}{|c|}{ Cruise 9701} \\
\hline 1 & 0.437 & $\mathrm{x}$ & & & & & & & & \\
\hline 2 & 0.435 & $x$ & & & & $\mathrm{x}$ & & & & \\
\hline 3 & 0.314 & $x$ & & $\mathrm{x}$ & & $\mathrm{x}$ & & & & \\
\hline 4 & 0.249 & $\mathrm{x}$ & & $\mathrm{x}$ & $\mathrm{x}$ & $\mathrm{x}$ & & & & \\
\hline 5 & 0.228 & $\mathrm{x}$ & $\mathrm{x}$ & $\mathrm{x}$ & $\mathrm{x}$ & $\mathrm{x}$ & & & & \\
\hline 6 & 0.175 & $\mathrm{x}$ & & $\mathrm{x}$ & $\mathrm{x}$ & $\mathrm{x}$ & $\mathrm{x}$ & $x$ & & \\
\hline 7 & 0.166 & $\mathrm{x}$ & $\mathrm{x}$ & $x$ & $\mathrm{x}$ & $\mathrm{x}$ & $\mathrm{x}$ & $\mathrm{x}$ & & \\
\hline \multicolumn{11}{|c|}{ Study total } \\
\hline 1 & 0.610 & $\mathrm{x}$ & & & & & & & & \\
\hline 2 & 0.519 & $\mathrm{x}$ & $\mathrm{x}$ & & & & & & & \\
\hline 3 & 0.498 & $\mathrm{x}$ & $\mathrm{x}$ & & $\mathrm{x}$ & & & & & \\
\hline 4 & 0.494 & $\mathrm{x}$ & $\mathrm{x}$ & & $x$ & & $\mathrm{x}$ & & & \\
\hline 5 & 0.479 & $\mathrm{x}$ & $x$ & & $x$ & & $\mathrm{x}$ & & & $\mathrm{x}$ \\
\hline 6 & 0.464 & $\mathrm{x}$ & $x$ & $\mathrm{x}$ & $x$ & & $\mathrm{x}$ & & & $\mathrm{x}$ \\
\hline 7 & 0.408 & $\mathrm{x}$ & $\mathrm{x}$ & $\mathrm{x}$ & $\mathrm{x}$ & & $\mathrm{x}$ & & $x$ & $x$ \\
\hline 8 & 0.387 & $\mathrm{x}$ & $\mathrm{x}$ & $\mathrm{x}$ & $\mathrm{x}$ & & $\mathrm{x}$ & $\mathrm{x}$ & $x$ & $x$ \\
\hline
\end{tabular}

were the variables that better fit the biological ordination. The $x$ axis in the biological ordination represents a depth gradient with shallow trawl stations (less than $50 \mathrm{~m}$ depth) clustering to the left and deep stations (more than $50 \mathrm{~m}$ depth) clustering to the right. As the $50 \mathrm{~m}$ depth limit can be taken to represent the border of the continental shelf in the Gulf of Salamanca (see Fig. 2 and Stromme \& Saeterdsal 1989) this result suggests the existence of 2 distinct assemblages: one belonging to the continental shelf and the other belonging to the upper slope.
Table 5. Percentage contributions of typifying species (over $5 \%$ ) and discriminating species (over $2 \%$ ) to within-group similarity and between-group dissimilarity, respectively, identified by SIMPER analysis examining between the 2 demersal fish assemblages found in the Gulf of Salamanca. S: shelf; U.SL.: upper slope

\begin{tabular}{|c|c|c|}
\hline Typifying species & S. $(<50 \mathrm{~m})$ & U.SL. (>50 m) \\
\hline Lutjanus analis & 31.77 & - \\
\hline Calamus penna & 19.54 & - \\
\hline Balistes capriscus & 14.79 & - \\
\hline Diodon holacanthus & 6.68 & - \\
\hline Selene vomer & 5.92 & - \\
\hline Chaetodipterus faber & 5.04 & \\
\hline Lutjanus synagris & 7.17 & 37.19 \\
\hline Rhomboplites aurorubens & - & 15.32 \\
\hline Ctenosciaena gracilicirrhus & - & 9.54 \\
\hline Pristipomoides aquilonaris & - & 9.40 \\
\hline Pagrus pagrus & - & 5.93 \\
\hline Sciaena trewavasae & - & 5.18 \\
\hline Within-group similarity & 29.29 & 14.83 \\
\hline Discriminating species & \multicolumn{2}{|c|}{ S. vs U.SL. } \\
\hline Lutjanus analis & \multicolumn{2}{|c|}{10.07} \\
\hline Balistes capriscus & \multicolumn{2}{|c|}{7.98} \\
\hline Calamus penna & \multicolumn{2}{|c|}{8.27} \\
\hline Lutjanus synagris & \multicolumn{2}{|c|}{6.26} \\
\hline Diodon holacanthus & \multicolumn{2}{|c|}{5.02} \\
\hline Sciaena trewavasae & \multicolumn{2}{|c|}{3.10} \\
\hline Pristipomoides aquilonaris & \multicolumn{2}{|c|}{2.48} \\
\hline Chaetodipterus faber & \multicolumn{2}{|c|}{2.27} \\
\hline Ctenosciaena gracilicirrhus & \multicolumn{2}{|c|}{2.23} \\
\hline Selene vomer & \multicolumn{2}{|c|}{3.26} \\
\hline Selene setapinnis & \multicolumn{2}{|c|}{2.00} \\
\hline Rhomboplites aurorubens & \multicolumn{2}{|c|}{2.00} \\
\hline Lutjanus jocu & \multicolumn{2}{|c|}{2.26} \\
\hline Between-group dissimilarity & \multicolumn{2}{|c|}{93.90} \\
\hline
\end{tabular}

The ordination of the 25 trawl stations exhibits a marginal relation with the bottom salinity, sampling date, northern and western coordinates as shown by the BIO-ENV analysis (Table 4). This result suggested that the composition and distribution of the demersal fish assemblages in the Gulf of Salamanca was weakly dependent upon seasonal changes (at least for our sampling dates) and the only spatial gradient of importance was the one associated with depth. The secondary importance level of other variables was confirmed when the BIO-ENV analysis per cruise was considered. In Table 4 it can be seen that variables like bottom salinity and temperature ranked always in second place or first in one case but in combination with the depth.

The analysis of similarity (ANOSIM) revealed that the demersal fish assemblage of the shelf differed in structure from the assemblage of the upper slope $(p<$ 0.05 ). Table 5 shows the species found by the SIMPER procedure to typify and discriminate the trawl station 
Table 6. Comparisons of the mean biomass, density, number of species, and Hill numbers 1 and 2 per trawl station between the 2 demersal fish assemblages found in the study and between cruises. Mean values \pm standard error. S.. shelf; U.SL.: upper slope

\begin{tabular}{|c|c|c|c|c|c|c|}
\hline & $\mathrm{S} .(<50 \mathrm{~m})$ & U.SL. (>50 m) & 9600 & 9601 & 9602 & 9701 \\
\hline Biomass (kg) & $87.50 \pm 15.24$ & $14.60 \pm 3.19$ & $82.36 \pm 25.87$ & $64.59 \pm 18.98$ & $86.20 \pm 34.10$ & $47.09 \pm 22.50$ \\
\hline Density (ind $/ 0.04381 \mathrm{~km}^{2}$ ) & $276.43 \pm 71.63$ & $135.58 \pm 73.98$ & $204.57 \pm 107.29$ & $398.98 \pm 92.29$ & $339.71 \pm 171.64$ & $102.25 \pm 41.06$ \\
\hline Number of species & $14.80 \pm 1.48$ & $12.17 \pm 3.63$ & $11.39 \pm 1.50$ & $22.4 \pm 1.50$ & $15.29 \pm 2.58$ & $11.28 \pm 2.63$ \\
\hline Hill 1 & $6.68 \pm 0.56$ & $4.62 \pm 0.75$ & $5.44 \pm 0.90$ & $7.39 \pm 0.87$ & $6.84 \pm 1.02$ & $5.31 \pm 1.00$ \\
\hline Hill 2 & $5.56 \pm 0.54$ & $4.14 \pm 1.06$ & $4.53 \pm 0.77$ & $6.03=1.11$ & $5.67 \pm 1.11$ & $4.78 \pm 0.98$ \\
\hline$n$ & 19 & 6 & 6 & 5 & 7 & 7 \\
\hline
\end{tabular}

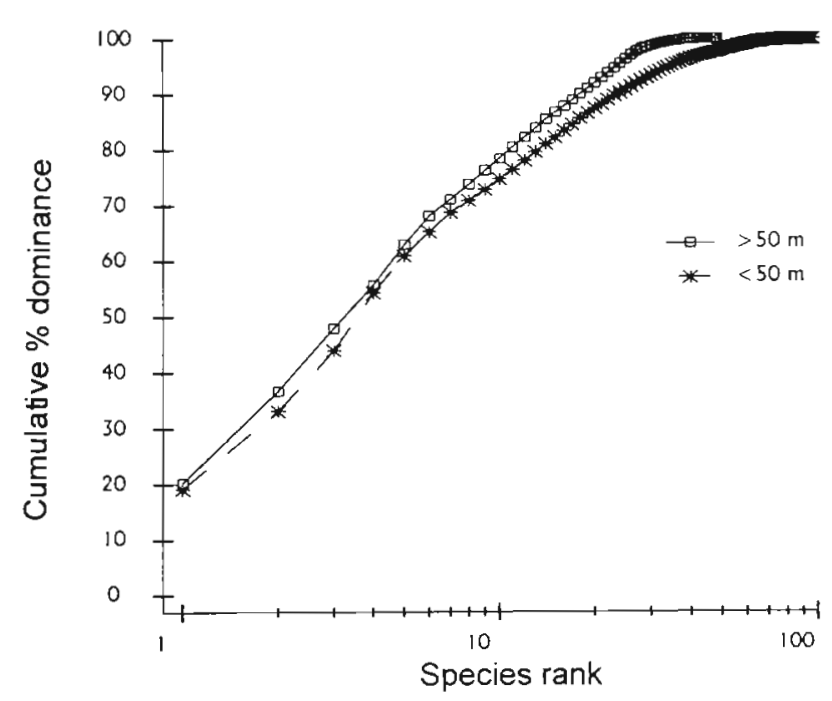

Fig. 6. Comparison of biomass dominance patterns between the continental shelf assemblage trawl stations and the continental slope assemblage trawl stations by means of $\mathrm{k}$-dominance curves

groupings according to the depth strata. Lutjanus synagris showed a general distribution and high abundance, and is important both as a typifying species inside the depth strata assemblages, although it typifies the upper slope assemblage particularly well (Table 5), and as a discriminating species. The discriminating species that ranked higher belonged to the shelf assemblage (Table 5), suggesting that their distribution was more restricted to their depth range than the distribution of the upper slope species.

Table 6 presents the mean values of biomass, density, species number, and Hill numbers 1 and 2 per trawl station by depth strata and for each cruise. The ANOVA showed that biomass per trawl station did differ between depth strata $(p<0.05)$, with the continental shelf showing a higher biomass concentration (Table 6). The ANOVA revealed no difference in the number of individuals per trawl station between depth strata as was also the case for number of species and Hill numbers 1 and $2(p>0.05)$.
Fig. 6 shows the k-dominance curves for both depth strata in the study based on biomass data. The shelf assemblage exhibited less dominance than the upper slope assemblage, i.e. in the shelf the distribution of the biomass among species is more even than in the upper slope.

The analysis of similarity (ANOSIM) between cruises by depth strata did not detect significant differences in assemblage structure $(p>0.05)$, i.e. the abundance, distribution and association patterns of the background species remained roughly the same in time, at least for the dates of the cruises.

The ANOVA revealed that biomass, density, and Hill numbers 1 and 2 per trawl station did not differ statistically between cruises ( $p>0.05)$, whereas the number of species per trawl station did $(\mathrm{p}<0.05)$. Tukey test showed that Cruise 9601 (April 1996, RV 'Malpelo') had significantly $(\mathrm{p}<0.05)$ more species per trawl (Table 6) than Cruise 9701 (February 1997, RV 'Ancon'). The other pairwise cruise comparisons were not significant $(\mathrm{p}>0.05)$.

\section{DISCUSSION}

While density and biomass values were standardized to area swept, the use of 2 different trawls in the survey left the number of species per trawl station as the main source of bias. Indeed, a significant difference was found between Cruises 9601 (RV 'Malpelo', with a mean swept area of $0.0701 \mathrm{~km}^{2}$ ) and 9701 (RV 'Ancon', with a mean swept area of $0.0327 \mathrm{~km}^{2}$ ) which were 10 mo apart, with Cruise 9601 having more species per trawl station. However, no other pairwaise comparison resulted in statistical differences, i.e. Cruise 9601 differed only from one of the $2 \mathrm{RV}$ 'Ancon' cruises and Cruise 9602 (RV 'Malpelo') was not different in species number from any of the other cruises. Thus, the results of the study seem to be not unduly influenced by the different fishing gears.

The presence-dominance pattern found here seems not to be uncommon in demersal fish communities. Similar pictures can be found, for instance, in Bianchi 
(1992b, c) and Wenner \& Sedberry (1989). As the resource availability in the benthos can be thought to be at a premium for the fish, there seems to be a strong hierarchical organization in the demersal fish assemblages where a few species tend to preempt wide use of the resources by the rest. On the other hand, the mechanisms responsible for the maintenance of the rich demersal fish species diversity (an average of 5 new species resulted in each tow) are worth investigating. Even though the sedimentary bottom may look structurally simple, the presence of Las Animas Bank, of mangrove forest on the coastline, the seasonal influence of continental run of and winds, and the oceanographic regime may explain this demersal fish species richness.

Two distinctive demersal fish assemblages clearly associated with the topography of the gulf emerge from the analysis: a shallow assemblage reaching the $50 \mathrm{~m}$ depth limit which represents the continental shelf, and a deep assemblage beyond that depth which represents the upper slope. The first assemblage exhibits greater biomass and contains species of larger size and commercial interest (for instance, Lutjanus analis and Calamus penna); the second one is characterized by the presence of species like Porichthys plectrodon, Sciaena trewavasae and Steindachneria argentea, which are small and non-commercial species, although the dominance of snappers of median size (L. synagris, Rhomboplites aurorubens and Pristipomoides aquilonaris) make it of interest for fishing (Table 5). The finding that biomass declines with depth is in accordance with reports elsewhere in the world (Darcy \& Gutherz 1984, Pauly 1988, Gonzalez-Sansón et al. 1997).

As shown in this study the main determining bottom feature associated with the structure of the demersal fish assemblages is the depth as it reflects the change from the continental shelf to the continental slope. Other bottom and oceanographic factors do play a role, however. Thus, with the exception of Cruise 9600 (December 1995) when grain size in combination to depth scores second in the BIO-ENV correlation, in the other 3 cruises bottom temperature and salinity (always in combination with depth) scored first or second, i.e. the distribution pattern of bottom salinity and temperature, for a given period, seems to show enough contrast to influence the configuration of the assemblages as has been detected in other studies in particular in relation to temperature (Bianchi 1991, 1992b, c, Gonzalez-Sansón et al. 1997).

As depth (topography), sediment grain size and organic content can be considered as fixed or very conservative features, assemblage temporal change must be associated with variables such as salinity and temperature as they are expected to show more temporal variability. The ANOSIM procedure, however, did not detect temporal change in the overall assemblage structure between cruises as was also the case, in general, with the ANOVA analysis of univariate community aspects. These findings suggested that temporal change in the assemblage composition was weakly associated with seasonal change, at least for our cruise dates. This is not to say that the assemblages did not change with time. The spatial patchiness is matched by temporal patchiness of a vast number of species (Table 3).

The dominance pattern found here partly corresponds to a scheme proposed by Longhurst (1969) for western tropical Africa. Longhurst (1969) suggested 3 demersal fish groups based on the dominant species. The first group occurred in warm estuaries or coastal clear waters above the thermocline, on muddy or sandy bottoms and was dominated by the family Sciaenidae; the second group appeared in colder waters under the thermocline, on hard or sandy bottoms and was dominated by the families Sparidae and Lut-

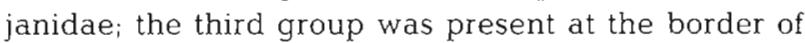
the continental shelf and beyond, and was dominated by merluccids and related species.

As samples from depths shallower than $10 \mathrm{~m}$ were not collected (see 'Materials and methods'), Longhurst's first group had no equivalent in this study. There is a coincidence between Longhurst's second group and the shelf assemblage as defined here, where Lutjanidae and Sparidae showed dominance, but partially so with Longhurst's third group (border of the continental shelf) as the upper slope assemblage in the Gulf of Salamanca is dominated by small snappers and sciaenids (Table 5), although Steindachneria argentea, a merlucid, was important in depths of more than $50 \mathrm{~m}$ in Cruise 9601 and a representative of the genus Synagrops ( $S$. trispinosus), one of the Longhurst's 'related species', also occurred in 2 deep stations.

The scheme proposed by Lowe-McConnell (1962) for the shelf off Guyana consisted of 'brown' (catfishes, rays), 'golden' (sciaenids), 'silver' (carangids, grunts) and 'red' (snappers) fish zones, from the shallow to the deep shelf. This scheme does not seem to fit the dominance pattern found in the Gulf of Salamanca. For instance, its 'red' fish zone is found between 80 and $120 \mathrm{~m}$ depth, whereas the snappers are typical species in the full depth range of this study (Table 5). The demersal fish assemblages in the Gulf of Salamanca would resemble a mixture of Lowe-McConnell's 'golden', 'silver' and 'red' fish zones.

Bianchi (1992a), by means of quantitative multivariate methods, described 12 assemblages for the shelf off Venezuela, Guyana and Surinam, 6 for the dry/windy climatic condition (May) and 6 for the rainy/calm cli- 
matic condition (August) which, as is the case here, primarily responded to a depth gradient. At the species level, in terms of dominance patterns, none of these 12 assemblages seems to correspond with the assemblages of the Gulf of Salamanca (compare Tables $7 \& 8$, p. 131 to 134, in Bianchi 1992a with Table 5 in this study), although both areas share many species. The same is true at the family level: in Bianchi's assemblages the families Sparidae and Balistidae are never of importance whereas their representatives typify the assemblages in the Gulf of Salamanca (Table 5). Representatives of the families Clupeidae and Trichiuridae are typifying species in Bianchi's (1992a) assemblages but not in this study. On the other hand, both studies have in common that representatives of the families Lutjanidae, Sciaenidae and Carangidae are among the most important typifying species in the assemblages.

Strong temporal change was a notorious feature in the assemblages described by Bianchi (1992a). The assemblages found in the May cruise got reorganized in the August cruise in terms of their dominance patterns and spatial coverage. Bianchi (1992a) attributes this to uneven sampling effort in the 2 cruises and to the migration of a number of species from deep waters to shallow waters and vice versa. This contrasts with the situation found in the Gulf of Salamanca where temporal change was not strong enough to alter the basic dominance pattern from one cruise to the next, although many species were very patchy in time (Table 3).

The comparison with the zonation schemes by Longhurst (1969) and Lowe-McConnell (1962) and with the assemblages of Bianchi (1992a) are obscured by the great difference in spatial scale. Our study area, including the non-trawlable area and the area less than $10 \mathrm{~m}$ depth (see 'Materials and methods'), is $955 \mathrm{~km}^{2}$ which, for instance, is 125 times smaller than the study area of Bianchi (1992a), which was $120000 \mathrm{~km}^{2}$, approximately (calculated from her Fig. 1, p. 119). A second great difference is the width of the continental shelf. While in Bianchi (1992a) the shelf spreads $180 \mathrm{~km}$ from the coast, in the Gulf of Salamanca the continental shelf just reaches to $16 \mathrm{~km}$ from the coast. A third difference refers to the sampling density. Whereas in this study, in the cruise with fewer stations (Table 2), a station was placed every $191 \mathrm{~km}^{2}$ on average, in Bianchi (1992a), in the cruise with more stations (May, 94 trawl stations), a station was placed every $1276 \mathrm{~km}^{2}$ on average.

The narrow shelf in the Gulf of Salamanca probably precludes a vertical zonation of the demersal assemblages, other than the zonation described in this study, i.e. shelf versus upper slope. The small area in the Gulf of Salamanca probably allows for the manifestation of the effects of local habitats, for instance, the Animas Bank, on the composition and dynamics of the demer- sal fish fauna, effects that are obscured in studies covering bigger areas. Thus it is an open question whether Bianchi's (1992a) demersal assemblages and their temporal change have an ecological meaning or whether they are an artifact of the low sampling density, i.e. if new surveys were to be conducted under the same conditions and time of the year would the same assemblages emerge? Likewise only repeated sampling in the Gulf of Salamanca will tell to what extent the demersal fish assemblages identified are predictable

Another general factor that makes comparisons and the search for general descriptions in demersal assemblages a difficult task is the fishery regime. In all the cases discussed here the shelves are subject to substantial fishery pressure, except the Gulf of Salamanca in depths more than $50 \mathrm{~m}$. Clearly there is a distortion of the natural condition by this mortality source which is difficult to assess.

It is of interest to note that Balistes capriscus, which belongs to the background species in the Gulf of Salamanca (Table 3), appeared also as dominant in the Gulf of Guinea, which is characterized by a strong upwelling (Bianchi 1992b). According to Longhurst \& Pauly (1987) and Bianchi (1992b) this species had increased its abundance since the seventies as a result of fishing of competitors. As mentioned, B. capriscus is to date of no commercial interest for fishermen in the Gulf of Salamanca and is discarded, which may contribute to its survival. An open question is then whether a similar situation to that found in the Gulf of Guinea may be occurring in the Gulf of Salamanca.

Among the background species we found Lutjanus analis, L. synagris and Calamus penna (Table 3), 3 of the more interesting fishes for commercial purposes in the Caribbean area. This may emphasize the importance of the Gulf of Salamanca as an area to be managed with special care. The case of Balistes capriscus, normally ignored by fishermen, may require the development of an educational campaign as this fish has a general acceptance and is consumed in various tropical American countries including Mexico and Venezuela.

The overall picture gained here on the demersal fish communities in the Gulf of Salamanca stresses once more the multispecies character of the tropical fisheries. Management effort should take into account this fact as the fishing taking place in the gulf is not species selective. On the other hand, the ecological interdependence should be assessed as the impact of exploitation and of potential management measures on the demersal fish assemblages is unclear.

Acknowledgements. This work was supported by COLCIENCIAS Grant No 2105-09-176-94 for the project 'Estudio ecológico pesquero de los recursos demersales del Golfo de 
Salamanca, Caribe colombiano. Estimación de la variabilidad de los componentes biológicos del sistema' We thank $C$. Fernandez and $G$. Moreno for critical review of the manuscript and the crews of the RV 'Malpelo'and RV 'Ancon' for their support. The comments by 3 anonymous referees helped to improve the manuscript. NB: Interested readers can obtain additional data directly from the authors. This is INVEMAR contribution no. 609 .

\section{LITERATURE CITED}

Allen L (1982) Seasonal abundance, composition, and productivity of the littoral fish assemblage in upper Newport Bay, California. Fish Bull 80(4):769-790

Ansari ZA, Chatterji A, Ingole BS, Sreepada RA, Rivonkar CU, Parulekar AH (1995) Community structure and seasonal variation of an inshore demersal fish community at Goa, west coast of India. Estuar Coast Shelf Sci 41: 593-610

Bartels CE, Price KS, Lopez MI, Bussing WA (1983) Occurrence, distribution, abundance and diversity of fishes in the Gulf of Nicoya Costa Rica. Rev Biol Trop 31(1):75-102

Blanchi G (1991) Demersal assemblages of the continental shelf and slope edge between the Gulf of Tehuantepec (Mexico) and the Gulf of Papagayo (Costa Rica). Mar Ecol Prog Ser 73:121-140

Bianchi G (1992a) Demersal assemblages of tropical continental shelves. A study based on the data collected through the surveys of the R/V 'Dr. Fridtjof Nansen' Dr Scient thesis, University of Bergen

Bianchi G (1992b) Demersal assemblages of the continental shelf and upper slope of Angola. Mar Ecol Prog Ser 81: $101-120$

Bianchi G (1992c) Study of the demersal assemblages of the continental shelf and upper slope off Congo and Gabon, based on the trawl surveys of the RV 'Dr Fridtjof Nansen' Mar Ecol Prog Ser 85:9-23

Blaber SJM, Brewer DT, Salini JP (1989) Species composition and biomasses of fishes in different habitats of a tropical northern Australian estuary: their occurrence in the adjoining sea and estuarine dependence. Estuar Coast Shelf Sci 29:509-531

Blanco J (ed) (1993) Reconocimiento piloto de fondos, ambiente, fauna asociada y recursos pesqueros en aguas costeras del Departamento del Magdalena. INVEMAR, Informe Final, Colciencias, Bogotà

Blanco J, Diaz J, Cortes M (1994) El Banco de las Animas: una amplia formación arrecifal desarrollada sobre un antiguo delta del río Magdalena. Bol Ecotróp 27:10-18

Buchanan JB, Kain JM (1984) Measurement of the physical and chemical environment sediment. In: Holme NA, McIntyre AD (eds) Methods for the study of marine benthos. IBP Handbook No. 16. Blackwell Scientific Publications, Oxford, p 41-65

Caddy JF, Sharp GD (1986) An ecological framework for marine fishery investigations. FAO Fish Tech Pap 283

Clarke KL (1993) Non-parametric multivariate analyses of change in community structure. Aust J Ecol 18:117-143

Clarke KL, Ainsworth M (1993) A method of linking multivariate community structure to environmental variables. Mar Ecol Prog Ser 92:205-219

Clarke KL, Green RH (1988) Statistical design and analysis for a 'biological effects' study. Mar Ecol. Prog Ser 46:213-226

Darcy GH, Gutherz EJ (1984) Abundance and density of demersal fishes on the West Florida Shelf. January 1978. Bull Mar Sci 34(1):81-107
Eslava J, Lopez V, Olaya G (1986) Contribución al conocimiento del régimen térmico y pluviométrico de Colombia. IGAC Col Geogr 12(2):53-118

Espino M, Wosnitza=Mendo C (1984) Manuales de evaluación de peces. \#1. Área barrida. Instituto del mar del Perú, Informe Tecnico 86

Field JG, Clarke KR, Warwick RM (1982) A practical strategy for analysing multispecies distribution patterns. Mar Ecol Prog Ser 8:37-52

González-Sansón G, Aguilar-Palomino A, Arciniegas-Florez J, Garcia de Quevedo R, Marchain E, Godinez-Dominguez V, Landa-Jaime J, Mariscal-Romero J, Michel-Morfin E, Saucedo-Lozano M (1997) Variación espacial de la abundancia de la fauna de fondos blandos en la plataforma continental de Jalisco y Colima. México (primavera 1995). Cienc Mar 23(1):93-110

Gray CA, Otway NM (1994) Spatial and temporal differences in assemblages of demersal fishes on the inner continental shelf off Sydney, South Eastern Australia. Aust J Mar Freshwat Res 45(4):665-676

Hill MO (1973) Diversity and evenness: a unifying notation and its consequences. Ecology 54(2):427-432

INPA (1995) Boletín estadístico pesquero. Instituto Nacional de Pesca y Acuicultura, División de Sistemas y Estadística

Lambshead PJ, Platt HM, Shaw KM (1983) The detection of differences among assemblages of marine benthic species based on an assessment of dominance and diversity. J Nat Hist 17:859-874

Longhurst AR (1969) Species assemblages in tropical demersal fisheries. Proc Sym Oceanogr Fish Trop Assoc, UNESCO, Paris, p 147-168

Longhurst AR, Pauly D (1987) Ecology of tropical oceans. Academic Press, New York

Lowe-McConnell RH (1962) The fishes of the British Guyana continental shelf, Atlantic coast of South America, with notes on their natural history. J Linn Soc Lond (Zool) 44 $669-700$

Márquez G (1982) Los sistemas ecológicos marinos del sector adyacente a Santa Marta. Caribe Colombiano I: Generalidades. Ecol Trop 2(1):5-18

Martin TJ, Brewer DT, Blaber SJ (1995) Factors affecting distribution and abundance of small demersal fishes in the Gulf of Carpentaria. Aust J Mar Freshwat Res 40(6): 909-920

Molina A (1993) Estudio sedimentológico y morfológico de la plataforma continental Caribe entre el sector de Santa Marta y Punta Morro Hermoso. Bol Cient $\mathrm{ClOH} \mathrm{14:67-77}$

Pauly D (1988) Fisheries research and the demersal fisheries of southeast Asia. In: Gulland JA (ed) Fish population dynamics. John Wiley, London, p 329-348

Pitcher A (1996) Reinventing fisheries management. NAGA ICLARM Q 19(3): 15-17

Rainer SF, Munro ISR (1982) Demersal fish and cephalopod communities of an unexploited coastal environment in northern Australia. Aust $J$ Mar Freshwat Res 33: $1039-1055$

Ramirez G (1993) Condiciones oceanográficas en el área del Golfo de Salamanca. In: Blanco JA (ed) Reconocimiento piloto de fondos, ambiente, fauna asociada y recursos pesqueros, en aguas costeras del Departamento del Magdalena. Informe Final, Colciencias, Bogotà, p 90-95

Salzwedel H, Müller K (1983) A summary of meteorological and hydrological data from the Bay of Santa Marta, Colombian Caribbean. An Inst Invest Mar Punta Betín 13: $67-83$

Sedberry RG, van Dolah RF (1984) Demersal fishes assemblages associated with hard bottom habitat in the South 
Atlantic of the USA. Environ Biol Fish 2(4):241-258

Stromme T, Saetersdal G (1989) Surveys of the fish resources in the shelf areas between Suriname and Colombia 1988. Reports on surveys with RV 'Dr. F. Nansen', Vol 1. Institute of Marine Research, Bergen

Watson RA, Dredge MLC, Mayer DG (1990) Spatial and seasonal variation in demersal trawl fauna associated with a prawn fishery on the Central Great Barrier Reef, Australia. Aust J Mar Freshwat Res 41(1):65-78

Wenner CA, Sedberry GR (1989) Species composition, distribution, and relative abundance of fishes in the coastal habitat off the southeastern United States. NOAA Tech

Editorial responsibility: Otto Kinne (Editor),

Oldendorf/Luhe, Germany
Rep NMFS 79:49

Wolff M (1996) Demersal fish assemblages along the Pacific coast of Costa Rica: a quantitative and multivariate assessment based on the Victor Hensen Costa Rica Expedition (1993/1994). Rev Biol Trop Suppl 3:187-214

Yañez-Arancibia A, Sánchez-Gil P (1986) Los peces demersales de la plataforma continental del sur de México. 1. Caracterización ambiental, ecológica y evaluación de las especies, poblaciones y comunidades. Inst Cienc del Mar y Limnol UNAM, México, Publicación especial 9, p 230

Zar JH (1984) Biostatistical analysis, 2nd edn. Prentice-Hall International lnc, Chichester

Submitted: February 19, 1998; Accepted: July 15, 1998

Proofs received from author(s): November 4, 1998 